\title{
Amigdalitis aguda recurrente bacteriana: Estudio prospectivo, comparativo y controlado de sus características clínicas y microbiológicas
}

\author{
Acute recurrent bacterial tonsillitis: Prospective, comparative \\ and controlled study of its clinical and microbiological characterisitics
}

\author{
Carolina Der $\mathrm{M}^{1}$, Rodrigo Iñiguez $\mathrm{C}^{2}$, Ana María Guzmán $\mathrm{D}^{3}$, David Jofré $\mathrm{P}^{2}$, \\ Armando Iñiguez $\mathrm{C}^{4}$, Jaime Labarca $\mathrm{L}^{5}$.
}

\begin{abstract}
RESUMEN
Introducción: La amigdalitis aguda recurrente es una patología de frecuente consulta, es una de las indicaciones de amigdalectomía. No se sabe con exactitud el origen de esta patología.

Objetivo: Identificar la microbiología y patrones de susceptibilidad de las bacterias en la amigdalitis aguda recurrente bacteriana (AARB) a los antimicrobianos más comúnmente en $\mathrm{d}$ medio nacional, usados en su tratamiento.

Material y método: Se planificó un estudio prospectivo, controlado y ciego. Se evaluaron pacientes (casos) con antecedentes de AARB con indicación quirúrgica Los criterios de inclusión de los casos fueron niños de ambos sexos entre 4 y 10 años, sin historia de inmunodeficiencias, no haber recibido antibióticos en los 15 días previos a lacirugía Se definió como "controles" a los pacientes con antecedentes de apnea del sueño con indicación quirúrgica y sin antecedentes de AARB o cuadros sugerentes de amigdalitis en los últimos 6 meses. En todos los pacientes se utilizó la misma técnica de toma de muestra de tejido para cultivo y antibiograma Para el estudio de sensibilidades se realizó un antibiograma de las cepas aisladas y se evaluó la presencia de beta lactamasas. Los datos fueron analizados con 1 programa S-plus para análisis univariado. Para evaluar proporciones se utilizó el test de chicuadrado o el test exacto de Fisher. Las variables continuas fueron evaluadas con el test de Wilcoxon Two sample.

Resultados: No se encontraron diferencias estadísticamente significativas entre las cepas encontradas en el grupo de casos y de controles. La bacteria aislada con más frecuencia fue Haemophilus influenzae en 26 muestras en cada grupo (81\%), seguido por $S$. Aureus y Streptococccus beta hemolítico grupo A(SBHGA). En cuanto a las asociaciones de patógenos más frecuentes, se encontró que las mayores asociaciones fueron $\mathrm{H}$. influenzae + S. aureus y H. influenzae + SBHGA Tampoco hubo diferencias esrtadísticamente significativas en frecuencia de estas asociaciones entre el grupo de SBHGA y los controles. En cuanto a la producción de betalactamasas se encontró que $7 \%$ de las cepas de Haemophilus influenzae de los pacientes con AARB, eran betalactamasas (+). En el grupo de pacientes con
\end{abstract}

${ }^{1}$ Médico del Servicio de Otorrinolaringología, Hospital Cínico Universidad de Chile.

2Médico del Servicio de Otorrinolaringología, Hospital Cínico Universidad Católica de Chile

${ }^{3}$ Médico del Servicio de Laboratorio Cínico, Hospital Cínico Universidad Católica de Chile

${ }^{4}$ Interno, Hospital Cínico Universidad Católica de Chile

${ }^{5}$ Médico del Servicio de Infectología, Hospital Cínico Universidad Católica de Chile 
hiperplasia amigdalina, $12 \%$ de las cepas de este microorganismo eran betalactamasa (+). Estas diferencias tampoco resultaron estadísticamente significativas $(p=1)$.

\section{ABSTRACT}

Background: The acute recurrent tonsillitis is a frequent pathology, and it is one of the indications of tonsillectomy. The origen of this pathology is not well known.

Aim: To identify the microbiology and susceptibility patterns of bacteria to antimicrobics of common use in acute recurrent bacterial tonsillitis (ARBT).

Methods: A prospective, controlled and blind study was planned. Patients ("Cases") with history of ARBT of surgical indication were evaluated. Inclusion criteria were the following: children of both sexes, between 4 and 10 years old, without immunodeficiency history, not receiving antibiotics for 15 days prior to surgery. "Controls" were defined as patients presenting with sleep apnea with surgical indication, without a history of ARBT or tonsillitissuggesting symptoms for the past 6 months. In all patients the same technique was used to obtain a tissue sample for culture and antibiogram. For the sensibility study an antibiogram of the isolated strains was carried out and the presence of beta lactamases was evaluated. The data was analyzed using the S-plus software for univariate analysis. To evaluate proportions, the chi-square or the Fischer test were used. Continue variables were evaluated with the Wilcoxon Two Sample test.

Results: There were no statistically significant differences in the strains found in Cases as compared to Controls. The most frequently found bacteria was Haemophilus influenzae in 26 samples of each group (81\%), followed by S. Aureus and SBHGA As to the more frequents pathogen associations, they were $H$. influenzae $+S$. aureus and $H$. influenzae+ SBHGA Similarly, there were no statistically significant differences in the frequence of these associations between the SBHGA group and Controls. As for beta lactamases production, it was found that $7 \%$ of the Haemophilus influenzae strains of ARBT patients were beta lactamases (+). Among patients with tonsil hyperplasia, $12 \%$ of this bacteria strains were beta lactamase (+). These differences were not statistically significant $(p=1)$.

\section{INTRODUCCIÓN}

La Amigdalitis Aguda Recurrente Bacteriana (AARB), mal Ilamada "amigdalitis crónica", porque no es un estado de continua infección, se define como un cuadro de amigdalitis bacteriana aguda que se repite con ciertafrecuenciaa pesar del tratamiento antibiótico. Además, desde el punto de vista de la práctica clínica y para efectos del presente trabajo, definimos amigdalitis bacteriana aguda como un cuadro de inicio brusco, odinofagia, fiebre alta, CEG, placas de pus en amígdalas, petequias en paladar blando, adenopatías cervicales, ausencia de tos y síntomas nasales u otros que puedan sugerir patología viral.
LaAARB es una patología frecuente y constituye una de las indicaciones de amigdalectomía ${ }^{1,2}$, la cirugía otorrinolaringológica más comúnmente practicada en Chile ${ }^{3}$. Los pacientes a los cuales se les indica tonsilectomía por esta causa son aquellos que han tenido múltiples diagnósticos de amigdalitis aguda bacteriana, con tratamientos antibióticos adecuados en cuanto a tipo y duración, dirigidos funda mentalmente a Streptococccus grupo A y que, no obstante, siguen presentando este cuadro $0^{4,5}$. Además, pacientes que a causa de esta patología presentan importante ausentismo laboral y escolar. Es así como se plantea el diagnóstico de AARB con indicación quirúrgica en aquellos casos que presen- 
ten 3 o más amigdalitis bacterianas al año por 3 años consecutivos; 05 o más en 2 años; 07 o más en 1 año, por el mayor riesgo de complicaciones no supurativas que implica dicha frecuencia?.

En cuanto a las bacterias involucradas en esta patología, en estudios previos realizados en el extranjero se ha encontrado una tasa significativamente mayor de recuperación de Haemophilus influenzaey Streptococccus beta hemolítico grupo A (SBHGA) en el centro amigdalino de pacientes con AARB, compa rado con controles normales ${ }^{6,7}$. Cabe destacar que los estudios mencionados carecen de análisis sistemático de resistencia antibióticay de los mecanismos involucrados en dicho proceso.

日 SBHGA se ha identificado como el principal patógeno en AARB, Haemophilus influenzae se ha asociado a la hiperplasia amigdalina y se cree que actuaría como un facilitador de la acción de SBHGA. Otros microorganismos identificados son $S$ aureus y $S$ Pneumoniae 8 .

Entre los estudios de susceptibilidad in vitro realizados en Chile en pacientes con amigdalitis aguda, se ha observado que Streptococcus grupo A presenta una susceptibilidad del $100 \%$ a penicilina, amoxicilina, amoxicilina-sulbactam y cefuroximo; $7,8 \%$ de resistencia a eritromicina; $0 \%$ a $5,2 \%$ de resistencia a azitromicina y $0 \%$ a $5,9 \%$ de resistencia a claritromicina ${ }^{9,10}$.

En el caso de macrólidos y lincosamidas se ha descrito que Streptococcus pyogenes produciría como principal mecanismo de resistencia una altera ción en el sitio blanco del antibiótico que se encuentra en la subunidad 50s del ribosoma, a través de la producción de metiltransferasas codificadas por el gen $\mathrm{QRM}$ de origen plasmidial o cromosomal que transformarían dicho sitio de acción ${ }^{9,11-14}$.

En el caso de Hemophilus influenzae, también existen publicaciones nacionales que describen su susceptibilidad in vitro en amigdalitis aguda, en donde se observó $15 \%$ a $30 \%$ de cepas beta lactamasa positivas y por lo tanto resistentes a penicilina ${ }^{15,16}$. Oro estudio muestra $12,8 \%$ de re sistencia a ampicilina para cepas de Hemophilus influenzae no capsulados aislados de pacientes con enfermedades respiratorias y $12,3 \%$ de resistencia a amoxicilina, siendo esta nula frente a amoxicilinasulbactam. Tampoco se ha observado resistencia a cefuroximo. En el caso de los macrólidos, se describe $9,1 \%$ de resistencia a claritromicina, sin observarse resistencia a azitromicina ${ }^{10}$.

日 mecanismo más común de resistencia a betalactámicos es mediante producción por parte del microorganismo de betalactamasas o alteración de la PBP (proteína de unión de penicilinas) ${ }^{8,11-13}$.

Ahora que conocemos el patógeno, sabemos que es sensible en la mayoría de los casos a beta lactámicos y podemos tratar adecuaday oportunamente los casos de amigdalitis aguda ¿Cómo se explica la recurrencia? Muchas teorías intentan explicar la recurrencia de las amigdalitis causadas por estos microorganismos, pese a un tratamiento antibiótico aparentemente adecuado; entre ellas destacan:

1) Factores del patógeno: a) aparición de cepas productoras de beta lactamasa en flora comensal, que inactivarían localmente el antibiótico impidiendo alcanzar concentraciones inhibitorias, favoreciendo la infección por Streptococcus pyogenes ${ }^{17}$; b) producción de beta lactamasas por parte de Hemophilus influenzae ${ }^{15}$; c) presencia de reservorios bacterianos dentro de las células del epitelio faríngeo que no serían alcanzados por algunos antibióticos ${ }^{18}$; e) reinfección con la misma o nuevas cepas ${ }^{15}$; f) desarrollo de toleranciabacteriana frente a los antimicrobianos ${ }^{19}$.

2) Factores del huésped: a) erradicación del Streptococcus viridans de la faringe, dejando un nicho factible de ser ocupado por Streptococcus grupo $\mathrm{A}^{20}$; b) baja adherencia a los tratamientos por parte de los pacientes ${ }^{21}$; c) inmunidad local inadecuada22,23; d) concentración insuficiente del antibiótico en el sitio de la infección ${ }^{24}$.

Sin embargo, ninguno de ellos ha demostrado un rol preponderante en esta patología.

Existen distintas líneas de investigación que intentan explicar larecurrencia, agrupándoseen aquellas que señalan que el antibiótico no alcanzaría concentración tisular suficiente, y otras en que habríaunapermanencia del patógeno en el tejido amigdalino. Respecto de esta última alternativa, Stjernquist-Desatnik señala que al cultivar tejido del centro amigdalino se aísla Streptococcus pyogenes y Haemophilus influenzaecon igual frecuencia en pacientes con hiperplasiay en casos de $\mathrm{AARB}^{25}$. Por otro lado, Ramírez ha encontrado un resultado diferente: Streptococcus pyogenes se aísla en el $47 \%$ de los pacientes con AARB v/s $10 \%$ en 
hiperplasia ${ }^{26}$. No existen publicaciones nacionales que evalúen frecuencia de aislamiento bacteriano y resistencia antibiótica en pacientes con AARB, por lo que planteamos el siguiente estudio.

\section{Objetivos}

1. Observar el tamaño amigdalino en pacientes pediátricos con indicación quirúrgica por AARB $\mathrm{v} / \mathrm{s}$ pacientes con apnea obstructiva del sueño.

2. Cuantificar y comparar la frecuencia de aislamiento de SBHGA y $H$ influenzae en tejido amigdalino de pacientes pediátricos con indicación quirúrgica por AARB v/s pacientes operados por apnea obstructiva del sueño.

3. Evaluar la susceptibilidad antibiótica in vitro de SBHGA y $H$ influenzae en ambos grupos a penicilina, cefalosporinas, y macrólidos y definir mecanismos de resistencia bacteriana.

4. Identificar el gen de resistencia mediantePCRen las cepas de SBHGA que resulten resistentes.

\section{MATERIAL Y MÉTODO}

Seplanificó un estudio prospectivo, controlado y ciego. Se evaluaron pacientes provenientes de los servicios de otorrinolaringología del Hospital Sótero del Río y del

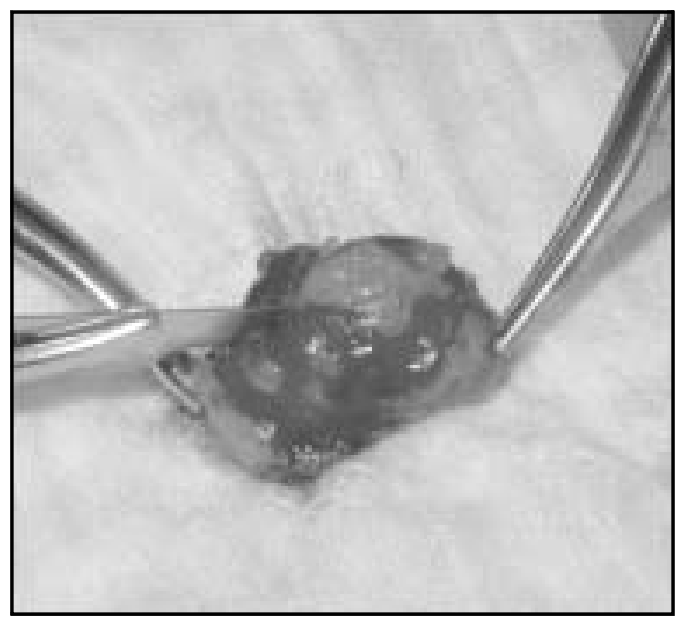

Figura 1. Procedimiento de apertura de la cápsula amigdalina para la posterior toma de muestra de tejido del centro amigdalino mediante técnica estéril.
Hospital Cínico de la de la Pontificia Universidad Católica entre enero y noviembre de 2002.

Para ingresar al estudio, el apoderado del paciente firmó el consentimiento informado y los criterios de inclusión fueron:

- Niños de ambos sexos entre 4 y 10 años

- Sin historia de inmunodeficiencias

- No haber recibido antibióticos en los 15 días previos a la cirugía

Se definió como "casos" a los pacientes con:

- Antecedentes de AARB con indicación quirúrgica

- Al menos 7 episodios el último año

- Concordancia en el diagnóstico de al menos 3 otorrinolaringólogos (1 no vinculado al estudio)

Se definió como "controles" a los pacientes con:

- Antecedentes de apnea del sueño con indicación quirúrgica

- Sin antecedentes de AARB o cuadros sugerentes de amigdalitis en los últimos 6 meses

En el pabellón de cirugía, se realizó amigdalectomía por disección o con Daniels tanto para casos como controles y se diseñó una técnica de toma de muestra de tejido a fin extraer el centro amigdalino sin contaminar la muestra Para ello se utilizó material estéril y mediante una incisión para la apertura de la cápsula amigdalina (Figura 1), se identificó y disecó el "core" o centro amigdalino (Figura 2), tejido que se transportó

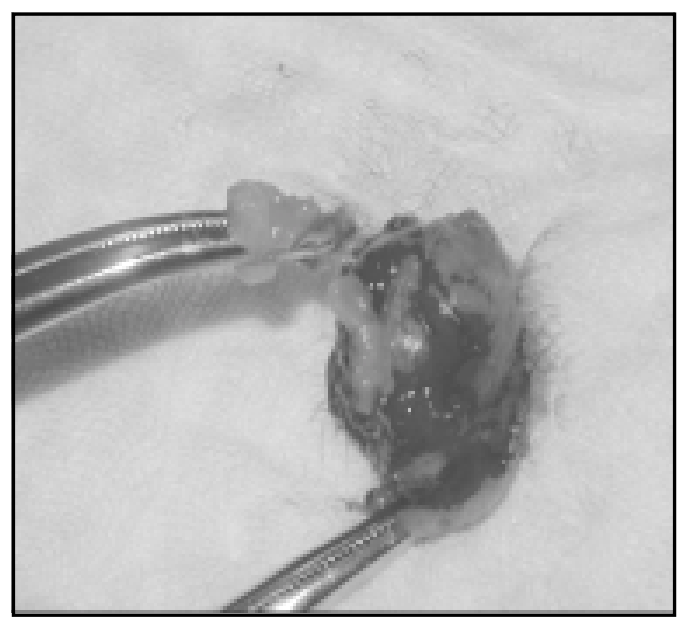

Figura 2. Procedimiento de toma de muestra de tejido del centro amigdalino mediante técnica estéril, tras la apertura de la cápsula amigdalina. 
en tubo estéril al laboratorio de microbiología, en donde se realizó un macerado del tejido, que posteriormente se sembró en agar sangre, agar chocolate y tioglicolato.

Para el estudio de sensibilidades se realizó un antibiograma de las cepas aisladas y se evaluó la presencia de beta lactamasas. Se agregó estudio mediante PCR para identificar genes de resistencia a antibióticos en aquellas cepas que resultaran resistentes a macrólidos, penicilinas y cefalosporinas.

Se calculó el tamaño de los grupos muestrales con un programa computacional que incluía pruebas de proporciones para grupos independientes. Se determinó que el número necesario para encontrar diferencias estadísticamente significativas, con un valor $p$ de 0,05 y una potencia de 0,8 fue de 30 pacientes en el grupo de casos y 30 pacientes en el grupo control.

Los datos fueron analizados con el programa S-plus para análisis univariado. Para evaluar proporciones se utilizó el test de chi-cuadrado o el test exacto de Fisher. Las variables contínuas fueron evaluadas con el test de Wilcoxon Two sample.

\section{RESULTADOS}

Se estudiaron las amígdalas de 33 pacientes con AARB y de 33 pacientes con hipertrofia adenoamigdalina. La edad promedio de ambos grupos fue de 6,1 y 5,9 años respectivamente. En cuanto a la distribución por género, el grupo de AARB estaba conformado por $40 \%$ de hombres y $60 \%$ de mujeres, mientras que en el grupo de hipertrofia adenoamigdalina, $40 \%$ de los casos eran mujeres y $60 \%$ hombres.

En relación al tamaño amigdalino, el grupo de AARB tenía $15 \%$ de las amígdalas grado I, 55\% de amígdalas grado II, $18 \%$ de amígdalas grado III y $12 \%$ deamígdalas grado IV. Entanto, el grupo control (hiperplasia amigdalina) presentó $42 \%$ deamígdalas grado III y $58 \%$ de amígdalas grado IV. Estas diferencias de tamaño amigdalino entreambos grupos fueestadística mente significativa con un valor $p=<0,0001$ (Tabla 1).

En cuanto a las bacterias aisladas, no se encontra ron diferencias estadísticamente significativas entre las cepas encontradas en el grupo de casos y decontroles. La bacteria aislada con más frecuencia fue Haemophilus influenzaeen 26 muestras en cada grupo (81\%), seguido por SAureus y SBHGA (Tabla2).

En cuanto a las asociaciones de patógenos más frecuentes, se encontró que las mayores asociaciones fueron Hinfluenzae + S aureus y Hinfluenzae + SBHGA. Tampoco hubo diferencias estadísticamente significativas en frecuencia de estas asociaciones entre el grupo de SBHGA y los controles (Tabla 3).

En la Tabla 4 se grafican las sensibilidades in vitro de los distintos gérmenes encontrados y las diferencias entre casos y controles. Para SBHGA,

Tabla 1. Tamaño amigdalino en grupo de casos y controles

\begin{tabular}{|lrrrr|}
\hline Tamaño amigdalino & Grado I & Grado II & Grado III & Grado IV \\
\hline Casos & $5(15 \%)$ & $18(55 \%)$ & $6(18 \%)$ & $4(12 \%)$ \\
Controles & 0 & 0 & $14(42 \%)$ & $19(58 \%)$ \\
\hline
\end{tabular}

Tabla 2. Bacterias aisladas en las muestras de casos y controles

\begin{tabular}{|lllllll|}
\hline Bacteria & H. influenzae & S. aureus & SBHGA & SBHGC & H. parainfluenzae & S. pneumoniae \\
\hline Casos & $26(81 \%)$ & $16(50 \%)$ & $6(18 \%)$ & $1(3 \%)$ & $2(6 \%)$ & $2(6 \%)$ \\
Controles & $26(81 \%)$ & $11(37 \%)$ & $10(31 \%)$ & $4(12 \%)$ & $2(6 \%)$ & 0 \\
Valor $p$ & $p=0,9$ & $p=0,3$ & $p=0,3$ & $p=0,2$ & $p=1$ & $p=0,4$ \\
\hline
\end{tabular}


Tabla 3. Combinaciones bacterianas aisladas en las muestras de casos y controles

\begin{tabular}{|lllll|}
\hline Asociación & $\begin{array}{l}\text { Hinfluenzaey } \\
\text { Saureus }\end{array}$ & $\begin{array}{l}\text { Hinfluenzaey } \\
\text { SBHGA }\end{array}$ & $\begin{array}{l}\text { Saureus } \\
\text { SBHGA }\end{array}$ & $\begin{array}{l}\text { Hinfluenzaey } \\
\text { SBHGC }\end{array}$ \\
\hline Casos & $13(41 \%)$ & $3(9 \%)$ & $1(3 \%)$ & $1(3 \%)$ \\
Controles & $8(25 \%)$ & $8(25 \%)$ & $3(9 \%)$ & $3(9 \%)$ \\
Valor $p$ & $p=0,3$ & $p=0,1$ & $p=0,3$ & $p=0,3$ \\
\hline
\end{tabular}

Tabla 4. Sensibilidad in vitro de las cepas aisladas en muestras de casos y controles

\begin{tabular}{|c|c|c|c|c|c|c|c|c|}
\hline \multirow[t]{3}{*}{ Patógeno } & \multicolumn{4}{|c|}{ Casos } & \multicolumn{4}{|c|}{ Controles } \\
\hline & \multicolumn{2}{|c|}{ B lactámicos } & \multicolumn{2}{|c|}{ Macrólidos } & \multicolumn{2}{|c|}{ B lactámicos } & \multicolumn{2}{|c|}{ Macrólidos } \\
\hline & $\mathrm{R}$ & $S$ & $\mathrm{R}$ & $S$ & $\mathrm{R}$ & $S$ & $\mathrm{R}$ & $S$ \\
\hline SBHGA & $0 \%$ & $100 \%$ & $0 \%$ & $100 \%$ & $0 \%$ & $100 \%$ & $0 \%$ & $100 \%$ \\
\hline SBHGC & $0 \%$ & $100 \%$ & $0 \%$ & $100 \%$ & $0 \%$ & $100 \%$ & $0 \%$ & $100 \%$ \\
\hline S. pneumoniae & $0 \%$ & $100 \%$ & $0 \%$ & $100 \%$ & - & - & - & - \\
\hline S. aureus & $0 \%$ & $100 \%$ & $13 \%$ & $87 \%$ & $0 \%$ & $100 \%$ & $0 \%$ & $100 \%$ \\
\hline
\end{tabular}

SBHGC y $S$ pneumoniae se encontró en todas las cepas aisladas una sensibilidad in vitro de $100 \%$ para betalactámicos y macrólidos. En el caso de $S$ aureus, se encontró $87 \%$ de sensibilidad a macrólidos y $100 \%$ de sensibilidad a betalactamicos en el grupo de casos y $100 \%$ de sensibilidad tanto a macrólidos como a betalactámicos en las cepas aisladas en el grupo de controles. Esta diferencia tampoco resultó estadísticamente significativa.

En cuanto a la producción de betalactamasas se encontró que $7 \%$ de las cepas de Haemophilus influenzae de los pacientes con AARB, eran betalactamasas (+). En el grupo de pacientes con hiperplasia amigdalina, $12 \%$ de las cepas de este microorganismo eran betalactamasa (+). Estas diferencias tampoco resultaron estadísticamente significativas $(p=1)$ (Tabla5).

No se realizó estudio de PCR para buscar genes de resistencia bacteriana en las cepas de SBHGA puesto que no se encontraron cepas resistentes.

\section{DISCUSIÓN}

En cuanto al tamaño amigdalino, es interesante resaltar que entre ambos grupos de estudio existen

Tabla 5. Cepas productoras de beta lactamasas en muestras de casos y controles

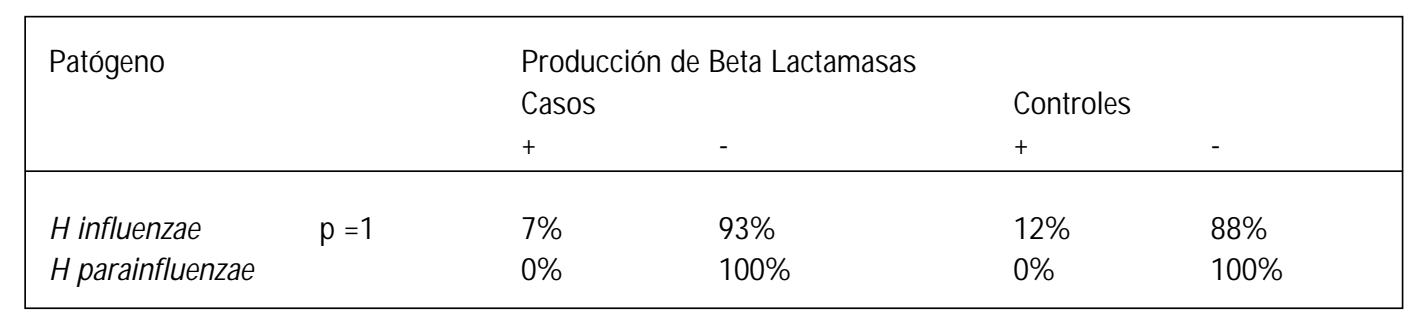


diferencias estadísticamente significativas: en el grupo con AARB, $30 \%$ de los pacientes presentó hiperplasia amigdalina, en cambio en el grupo de controles el $100 \%$ de ellos presentaba esta condición.

En ambos grupos, $81 \%$ de las amígdalas cultivadas tenía presencia de Haemophilus influenzae. No encontramos asociación estadística entre tamaño amigdalino y presencia de Haemophilus influenzae $(p=0,5)$.

En relación a los patógenos aislados y otros trabajos publicados, nuestros resultados difieren marcadamente deestudios españoles (Ramírezy cols), siendo más comparables al estudio noruego realizado por Stjernquist-Desatnik, en el que se señala que no existiría una diferencia en la frecuencia de aislamiento de SBHGA y Haemophilus influenzae entre pacientes con hiperplasia amigdalina y pacientes con AARB.

\section{CONCLUSIONES}

La recuperación de microorganismos desde el núcleo amigdalino es semejante (número, especies y resistencia antibiótica) tanto en pacientes pediátricos operados por AARB como en aquellos operados por apnea del sueño.

La persistencia de SBHGA en el tejido del centro amigdalino de pacientes con indicación quirúrgica por AARB no explica la recurrencia.

Se descarta el rol de $\mathrm{H}$ influenzae como causante de la recurrencia de SBHGA a través de la producción de beta lactamasas así como de generador de hiperplasia amigdalina.

Creemos que es necesario realizar más estudios para definir la etiología de la amigdalitis aguda recurrente bacteriana.

\section{Agradecimientos}

A los residentes del Servicio de Otorrinolaringología de la Pontificia Universidad Católica de Chile.

A los médicos staff del Servicio de Otorrinolaringología del Hospital Dr. Sótero del Río, en especial Dr. Iñíguez, Dra Salin y Dra. Larraguibel.

Trabajo financiado por la SOCHIORL en el concurso de proyectos de investigación de 2001 y presentado en el LIX Congreso Chileno de Otorrinolaringología y Grugía de Cabeza y Cuello.

\section{BIBLIOGRAFÍA}

1. BOAT T Y COLs. Tonsils and adenoids. In: Behrman RE, Vaughn VC III (eds). Nelson textbook of pediatrics. 12th ed. Philadelphia WB Saunders, 1983; 1019-22.

2. PARADISE JL Y coss. Efficacy of tonsillectomy for recurrent throat infection in severely affected children. The New England Journal of Medicine 1984; 310: 674-83.

3. CONTREAS JM. Adenomigdalectomía Rev Otorrinolaringol Or Cabeza Quello 1999; 59: 111.

4. Markowitz M, Graber MA, KaPLAN 且. Treatment of streptococcal pharyngotonsilitis: re ports of penicilin's demise are prematures. $J$ Pediatr 1993; 123: 679-85.

5. ÓsterLUND A Y cols. Intracellular reservoir of streptococcus pyogenes in vivo: a possible explanation for recurrent pharyngotonsillitis. Laryngoscope 1997; 107: 640-7.

6. Stuernauist-DesatNIK A, PrenlNer K, Sohalen C. High recovery of Haemophilus influenzae and group A estreptococci in recurrent tonsilar infection or hypertrophy as compared with normal tonsils. The journal of Laryngology and Otology 1991; 105: 439-41.

7. StJernaUIST-DESATNIK A, Hast E Tonsillar microbial flora: comparison of recurrent tonsillitis and normal tonsils. Acta Otolaryngol (Norway) 1999; 119(1): 102-6.

8. BRODSKY L Y cass. The role of Hemophilus influenzae in the pathogenesis of tonsillar hypertrophy in children. Laryngoscope 1988; 98(10): 1055-60.

9. VINAGRE Del P C Y cols. Emergencia de resistencia a macrólidos en Streptococcus pyogenes. Rev Méd Chile 1999; 127 : 1447-52.

10. PRADO V. Actividad comparativa in vitro de la combinación de amoxicilina-sulbactam y otros 4 antimicrobianos frente a bacterias aisladas de pacientes con infecciones respiratorias adquiri- 
das en la comunidad. Rev Chilena de Infectología 1997; 14(1): 28-38.

11. LeQleroQ R, COURVALIN P. Bacterial resistance to macrolide, lincosamides and streptogramin antibiotics by target modification. Antimicrob Agents Chemother 1991; 35: 1267-72.

12. ARTHUR M, MOLINAS G, MabiLAT G, COURVALIN P. Detection of erythromycin resistance by the polymerase chain reaction using primers in conserved regions of erm rRNA methylase genes. Antimicrob Agents Chemother 1990; 34(10): 2024-26.

13. ALBRTSS BRUCE Molecular biology of the cell 1994; 316-7.

14. SCHALEN C Y COLS. Characterization of an erytrhomycin resistance ( $\mathrm{EM}$ ) plasmid in streptococcus pyogenes. APMIS 1995; 103(1): 59-68.

15. PRADO V. Características fisiológicas y patoge néticas de Hemophilus influenzae. Sus proyecciones clínicas y terapéuticas. Rev Chilena de Infectología 1984; 2: 65-9.

16. TRUCOO OY COLS. Actividad in vitro de ampicilina y ampicilina-sulbactam sobre diversas bacterias. Rev Méd Chile 1989; 117: 747-54.

17. BROOK I. The role of $B$ lactamase producing bacteria in the persistence of streptococcal tonsillar infeccion. Rev Infect Dis 1984; 6: 601-7.

18. GRAHN E, HOLM SE, RoOs K. Penicillin tolerance in beta-streptococci isolated from patients with tonsillitis. Scand J Infect Dis 1987; 9: 421-6.
19. LAl CJ, WeSBLUM B. Altered methylation of ribosomal RNA in an erythromycin-resistant strain of Staphylococcus aureus. Proc Natl Acad Sci USA 1971; 68: 856-60.

20. Roos K Y cols. Interfering alpha estreptococci as a protection against recurrent tonsillitis in children. Int J Pediatr Airhinolaryngol 1993; 25: 141-8.

21. GR円N JL, RAY SP. Recurrence of streptococcal pharynngitis related to oral penicillin. $J$ Pediatr 1969; 75: 292-4.

22. KoOH RJ, BRODSKY L. Effect of specific bacteria on limphocyte proliferation in deseased and nondiseased tonsils. Laryngoscope 1993; 103 : 1020-6.

23. KoOH RJ, BRODSKY L. Qualitative and quantitative inmunoglobulin production by specific bacteria in chronic tonsillar disease. Laryngoscope 1995; 105: 42-8.

24. Roos K Y coss. Pharmacokinetics of phenoxymethylpenicillin in tonsils. Scand J Infect Dis 1986; 18: 125-30.

25. STJERNQUIST-DESATNIK A. Acta Otolaryngol (Norway) 1990; 109(3-4): 314-9.

26. RAMíREZ A Y cols. Beta hemolytic streptcocci in tonsil hypertrophy and recurrent tonsiIlitis. Enferm Infecc Microbiol Clin 1997; 15: 315-8. 